\title{
O Mecanismo de Transmissão Monetária na Economia Brasileira Pós-Plano Real*
}

\author{
Marcelo Fernandes ${ }^{* *}$ \\ Juan Toro ${ }^{* * *}$
}

Sumário: 1. Introdução; 2. Considerações metodológicas; 3. Modelo Estatístico; 4. Ajustes de curto prazo; 5. Conclusão.

Palavras-chave: análise de cointegração; curva IS; determinação de reservas; política monetária; regra de reação do Banco Central.

Códigos JEL: C32; E4; E5; F32.

Este trabalho estima o mecanismo de transmissão monetária da economia brasileira pós-Plano Real via um modelo VAR cointegrado para o estoque real de moeda, renda real agregada, inflação, e taxas de juros de curto e longo prazo. Para dar uma conotação estrutural ao sistema dinâmico estimado, identificamos os vetores de cointegração a partir de relações econômicas de equilíbrio. Os resultados empíricos evidenciam que o estoque real de moeda é uma tendência estocástica comum do sistema e que existem três relações de longo prazo. A primeira corresponde a uma curva IS estabelecendo uma relação negativa entre o hiato do produto real e a taxa de juros real. O segundo vetor de cointegração determina o nível de reservas em função da velocidade da moeda, da inflação e das taxas de juros de curto e longo prazo. A terceira relação identifica a regra de reação monetária do Banco Central para fixar a taxa de juros SELIC. Finalmente, a dinâmica de curto prazo implícita na representação VECM e a análise de impulso-resposta ilustram a boa aderência do modelo.

This paper estimates the monetary transmission mechanism in Brazil as from the Real Plan using a cointegrated VAR model for real money stock, real output, inflation, short- and long-term interest rates. To provide a structural interpretation to the resulting dynamic system, we identify the cointegrating vectors through

\footnotetext{
${ }^{*}$ Artigo recebido em dez. 2000 e aprovado em dez. 2003. Este trabalho faz parte de um projeto financiado pela Secretaria de Política Econômica do Ministério da Fazenda e pelo PRONEX. Os autores são extremamente gratos a dois pareceristas anônimos, Sergio Ribeiro da Costa Werlang e Joaquim Levi por comentários muito construtivos. Qualquer erro é de exclusiva responsabilidade dos autores.

${ }^{* *}$ Escola de Pós-Graduação em Economia, Fundação Getulio Vargas. E-mail: mfernand@fgv.br

${ }^{* * *}$ Department of Economics, University of Oxford e CentrA. E-mail: juan.toro@fundacioncentra.org
} 
equilibrium relationships derived from macroeconomic theory. The empirical results evince that the real money stock is a stochastic common trend of the system and that there are three long-run relationships. The first corresponds to the IS curve, establishing a negative relation between the real output gap and the real interest rate. The second cointegrating vector determines the level of international reserves as a function of money velocity, inflation, short- and long-term interest rates. The third cointegrating relationship identifies the monetary reaction rule that the Brazilian Central Bank uses to set the target interest rate (SELIC). Finally, the short-run dynamics implied by the VECM representation and the results of the impulse-response analysis demonstrate that the model fits well the data.

\section{Introdução}

O processo hiperinflacionário após a derrocada do Plano Cruzado culminou em uma série de planos de estabilização nos anos 80, normalmente calcados em fortes intervenções estatais e congelamentos de preços. Após a liberalização financeira e comercial promovida no início dos anos 90, o Plano Real trouxe uma redução drástica da taxa de inflação. Com efeito, a inflação caiu de um patamar anual draconiano de mais de $5.000 \%$ em junho de 1994 para algo em torno de $4 \%$ em meados de 1998. Na ausência do imposto inflacionário, a renda real brasileira cresceu substancialmente, enquanto que a redução do nível de incerteza estimulou a revitalização da atividade econômica até o advento da crise asiática em 1997 (ver, por exemplo, Camargo et alii (2002)). Ademais, as recentes mudanças na política monetária praticada pelas autoridades brasileiras são evidentes.

Apesar de existirem diversos mecanismos de transmissão monetária, dois canais são fundamentais, a saber, taxas de juros e câmbio. O primeiro canal surge da relação negativa entre o valor presente de bens duráveis e a taxa de juros real, uma vez que essa última mensura, de certa forma, a eficiência marginal do capital. Uma taxa de juros mais baixa estimula a produção de bens duráveis e, via multiplicador, também aumenta a demanda agregada. Apesar da importância do canal de transmissão via taxa de câmbio no controle da inflação, a acumulação substancial de reservas internacionais em um ambiente de taxas de juros elevadíssimas ilustra a ancora monetária implícita no processo de estabilização da economia brasileira. O processo de estabilização vem recuperando paulatinamente os demais mecanismos de transmissão monetária (via crédito e riqueza, por exemplo), de forma a mitigar a necessidade de taxas de juros tão elevadas. Afinal, em um regime de 
câmbio flutuante, uma política monetária pode ser conservadora mesmo com taxas de juros moderadas.

Esse artigo objetiva estimar o mecanismo de transmissão de política monetária da economia brasileira pós-Plano Real através de uma abordagem multivariada. Mais precisamente, estimamos um modelo vetor auto-regressivo (VAR) cointegrado para oferta monetária, renda agregada, preços, e taxas de juros de curto e longo prazo. A motivação reside em caracterizar os efeitos de curto e longo prazo, além das possíveis interações entre as variáveis que compõe o sistema. Para tal, utilizamos o conceito de cointegração para definir as relações econômicas de equilíbrio a partir de restrições de identificação.

Seguindo a metodologia proposta em Juselius (1998), determinamos o modelo empírico em dois estágios. Primeiro, estimamos por (pseudo) máxima verossimilhança as tendências estocásticas comuns, procurando identificá-las e interpretá-las à luz da teoria econômica. Segundo, construímos um modelo de correção de erros, que apresenta a propriedade de reversão aos equilíbrios de longo prazo. Deste modo, o modelo nos permite investigar as interações de curto prazo entre as variáveis do sistema e a velocidade de ajuste às relações de equilíbrio. A figura 1 resume a metodologia econométrica destacando as três fontes de informação: teoria econômica, aspectos institucionais, e as propriedades estatísticas das séries temporais.

O restante do artigo está assim organizado. A seção 2 discute os aspectos metodológicos do arcabouço econométrico e descreve as relações de cointegrações esperadas à luz da teoria monetária moderna. A seção 3 define o modelo estatístico e suas características empíricas. Em particular, os resultados mostram que o processo inflacionário é integrado e, portanto, não estacionário. Mostramos, em seguida, como evitar um modelo $I(2)$ considerando um sistema dinâmico composto por séries em termos reais (em vez de nominais) e taxa de inflação. Concluímos a seção 3 identificando empiricamente as relações estruturais de longo prazo. A seção 4 apresenta, então, um modelo dinâmico de correção de erros que explicita as características de longo e curto prazo do sistema. Finalmente, a seção 5 resume os principais resultados empíricos e tece algumas considerações finais.

\section{Considerações Metodológicas}

Assume-se em geral que o banco central dispõe de instrumentos para controlar a taxa de inflação e, possivelmente, influenciar tanto o nível de crescimento real da economia como a paridade de poder de compra. O mecanismo de transmissão monetária descreve a relação causal entre a intervenção monetária e as metas 
monetárias finais através dos instrumentos de política monetária. O controle monetário, seja direto ou indireto, depende da existência de uma conexão direta entre os níveis de estoque monetário e de preços. O controle do processo inflacionário especifica, então, uma meta monetária com limites superiores e inferiores.

No caso brasileiro, a meta monetária do Banco Central era, até 1998, o estoque de moeda medido pelo $M 3$. Adotou-se, então, um regime de metas inflacionárias, de modo que o estoque de moeda passou a ser inteiramente determinado pela demanda por moeda dos agentes. Em resumo, o mecanismo de transmissão monetária descreve como mudanças nos instrumentos de política monetária - excesso de moeda e taxa de juros de curto prazo - afetam a dinâmica da economia via taxa de juros de longo prazo, hiato do produto, e taxa de inflação.

Estimamos o mecanismo de transmissão a partir da metodologia empregada por Juselius (1998) calcada em um modelo VAR cointegrado. As principais variáveis relacionadas à política monetária possuem normalmente raiz unitária, tornando a análise de cointegração indispensável no contexto multivariado. A análise de cointegração permite a identificação estrutural das relações de longo prazo a partir de testes de restrições implicadas pela teoria econômica. Um exemplo clássico é a propriedade de homogeneidade de longo prazo dos preços, que valida a transformação de uma variável nominal em real.

O conjunto de variáveis relevantes é, infelizmente, demasiado extenso para uma análise empírica de equilíbrio geral. Os métodos de cointegração se encaixam perfeitamente nesse contexto de análise parcial, pois a propriedade de cointegração é invariante ao aumento do conjunto de informação. Portanto, no processo de seleção das variáveis seguiremos um procedimento que parte do específico para o geral, procurando investigar relações de equilíbrio entre um número restrito de variáveis antes de analisar o sistema por completo. No processo de escolha do modelo estatístico, utilizaremos a estratégia inversa para minimizar o erro de especificação, ou seja, do geral para o específico (ver, por exemplo, Garrat et alii (2000)).

A partir de um modelo VAR inicial, utilizaremos as propriedades de integração e cointegração dos dados para inferir as relações que determinam a eficácia do mecanismo de transmissão monetária, a saber

1. O grau de associação entre os instrumentos monetários de intervenção, as metas intermediárias, e a meta monetária final.

2. A dinâmica do ajuste de curto prazo resultante das variações nos instrumentos monetários e nas variáveis macroeconômicas relevantes. 
3. Os impactos de curto e longo prazo na inflação decorrente de choques na taxa de juros de curto prazo.

Considerando o número limitado de observações mensais, utilizaremos o conceito relativo de longo prazo sugerido por Juselius (1999). Interpretaremos uma relação de longo prazo como simplesmente uma relação de cointegração entre variáveis $I(1)$ e/ou $I(2)$. De forma análoga, a noção de ajustes de curto prazo indicará a reação de variáveis estacionárias às relações de cointegração e demais variáveis estacionárias. Uma condição necessária para a relevância de uma relação de longo prazo é, portanto, que exista ao menos um ajuste de curto prazo correspondente no sistema. Essa definição é muito conveniente na medida que qualquer relação de não cointegração incorretamente incluída entre as relações de longo prazo tende a ser desmascarada, uma vez que variáveis estacionárias não podem ajustar significativamente a variáveis não estacionárias.

\subsection{Relações esperadas de equilíbrio}

Até 1998, o Banco Central procurava controlar a inflação através da redução do nível agregado de liquidez na economia. Entretanto, considerando a liberalização financeira efetuada no início dos anos 90, a relação entre a liquidez da economia e o processo inflacionário pode também envolver as demandas por moeda de natureza transacional, precaucional e especulativa dos agentes. Assumindo homogeneidade de longo prazo tanto dos preços como da renda, chegamos a seguinte relação hipotética de demanda por moeda

$$
m_{t}-p_{t}-y_{t}^{r}=b_{0}-b_{1}\left(\pi_{t}-R_{t}^{d}\right)-b_{2}\left(R_{t}^{b}-R_{t}^{d}\right)+u_{t}^{m}
$$

na qual $b_{1}>0, b_{2}>0, m_{t}$ é o estoque monetário medido pelo M3, $y_{t}^{r}$ denota o produto real, $p_{t}$ é o índice de preços ao consumidor, $\pi_{t} \equiv \Delta p_{t}$ indica a taxa de inflação, e $u_{t}^{m}$ denota um processo estacionário $I(0)$ de memória curta.

A diferença entre as taxas de longo e curto prazo $R_{t}^{b}-R_{t}^{d}$ objetiva captar o custo relativo de oportunidade entre manter moeda (conceito M3) em vez de um título de longo prazo. ${ }^{1}$ O Banco Central exerce um controle indireto sobre o nível de liquidez agregada usando a taxa de juros de curto prazo para influenciar a demanda por moeda (1). Para que esse controle indireto funcione, algumas condições devem ser respeitadas. Primeiro, existe uma ligação direta entre o instrumento de intervenção do Banco Central e a meta intermediária. Segundo, uma variação na taxa de juros

\footnotetext{
${ }^{1}$ Nesse trabalho, utilizaremos letras minúsculas e maiúsculas para denotar variáveis em logaritmo e em nível, respectivamente.
} 
praticada pelo mercado afeta a demanda por moeda ou, pelo menos, o excesso de liquidez na economia. Terceiro, uma queda no estoque de moeda ou na demanda agregada resulta em uma queda correspondente nos preços.

A primeira condição refere-se ao canal de transmissão monetária via a taxa de juros fixada pelo Banco Central (ver Juselius (2001)). Essa condição é aparentemente satisfeita no caso brasileiro, uma vez que a taxa SELIC do Banco Central é reconhecidamente o principal determinante das taxas de juros praticadas pelo mercado. A segunda condição está diretamente relacionada com as propriedades de integração e cointegração dos dados. Se a hipótese das expectativas valer e $R_{t}^{b}-R_{t}^{d} \sim I(0)$, o controle monetário indireto não será muito eficaz, pois mesmo que $R_{t}^{d}$ suba após uma intervenção monetária, $R_{t}^{b}$ se ajustará proporcionalmente resultando em uma variação apenas marginal do custo de oportunidade $R_{t}^{b}-R_{t}^{d}$. Por outro lado, se $\pi_{t}-R_{t}^{d} \sim I(0)$ em função da paridade de Fisher, um aumento de $R_{t}^{d}$ resulta em um ajuste correspondente da taxa de inflação. Desta forma, a eficácia do controle monetário indireto depende essencialmente das propriedades da estrutura à termo das taxas de juros e da validade empírica da paridade de Fisher. A terceira condição exige implicitamente a propriedade de homogeneidade dos preços valha no longo prazo e requer que um choque permanente no estoque de moeda possua um impacto de longo prazo positivo nos preços.

Naturalmente, a eficácia das regras de política monetária ainda depende de dois fatores. Primeiro, que a variação da taxa de juros fixada pelo Banco Central afete, via a taxa de juros de curto prazo praticada pelo mercado, a taxa de juros de longo prazo. Em outras palavras, os choques nas taxas de juros de curto prazo determinam a taxa de juros de longo prazo. Segundo, um aumento da taxa de juros de longo prazo esfria a pressão do lado da demanda agregada na economia via níveis de consumo e investimento de longo prazo. Portanto, espera-se que a existência de um ajuste do produto à curva IS de longo prazo

$$
y_{t}^{r}-a_{1} t=a_{0}+a_{2}\left(\pi_{t}-R_{t}^{b}\right)+u_{t}^{y}
$$

em que $a_{1} \geq 0, a_{2}>0$, o produto real descontado por uma tendência linear $y_{t}^{r}-a_{1} t$ procura mensurar o hiato do produto, e $u_{t}^{y}$ consiste em um processo estacionário de memória curta $I(0)$. Por outro lado, se o hiato do produto estiver associado a uma inflação de demanda via uma curva de Phillips de curto prazo, então uma redução do hiato do produto via aumento da taxa de juros de longo prazo vai gerar uma menor inflação.

Uma relação alternativa particularmente adequada para o caso brasileiro envolveria também variáveis relacionadas ao Balanço de Pagamentos (ver Carneiro e Wu (2004)). Desde o início do Plano Real até janeiro de 1999, a utilização da taxa 
de câmbio como âncora nominal tornou o nível de atividade da economia brasileira mais exposto a choques externos. Tendo em vista a paridade descoberta das taxas de juros, variações no prêmio de risco são completamente absorvidas pela taxa de juros em um regime de taxa de câmbio fixa. Dessa forma, a taxa de juros nominal sinaliza a confiança na desinflação via efeitos do câmbio sobre a taxa de inflação esperada e, conseqüentemente, o hiato do produto passa a depender das contas externas. Nessas circunstâncias, taxas de juros elevadas desempenham um papel importante no esforço de financiamento do Balanço de Pagamentos atraindo capitais de arbitragem. O condicionamento das taxas de juros às necessidades de financiamento externo implica a subordinação dos juros às pressões de choques externos sobre o Balanço de Pagamentos.

Carneiro e Wu (2004) propõem utilizar uma Regra de Taylor modificada à la Clarida et alii (1998) para captar esse efeito. Nesse contexto, a taxa de juros de curto prazo reage não somente aos choques de oferta e ao hiato de produto, como também às variações no nível de reservas internacionais. Temos, então, a seguinte relação

$$
R_{t}^{d}=d_{0}+d_{1}\left(y_{t}^{r}-a_{1} t\right)+d_{2} \pi_{t}-d_{3} \Delta r_{t}+u_{t}^{r}
$$

na qual $r_{t}$ denota o logarítmico das reservas internacionais (conceito liquidez internacional) e $u_{t}^{r}$ um processo estacionário de memória curta $I(0)$. Essa especificação não se mostrou robusta empiricamente, de modo que tivemos que acrescentar a variação do câmbio (ver seção 4).

Para elaborar o modelo estatístico, precisamos estabelecer as propriedades de integração de cada variável. Os testes usuais de raiz unitária (Dickey e Fuller, 1979, Phillips e Perron, 1988) são, entretanto, muito sensíveis à presença de valores atípicos (Franses e Haldrup, 1994, Cati et alii, 1999). Como as séries em questão sofreram algumas intervenções no período pós-Plano Real, utilizamos o teste KPSS desenvolvido por Kwiatkowski et alii (1992). O teste KPSS inverte a hipótese nula checando a ausência (em vez de presença) de raiz unitária. A presença de valores atípicos prejudica assim apenas o poder do teste, não interferindo no seu tamanho. A rejeição da hipótese nula de estacionariedade possui, então, um significado ainda mais forte quando valores atípicos podem estar presentes.

Consideramos, então, o conjunto de variáveis $\left(\pi, y^{r}, m-p, R^{d}, R^{b}\right)$, no qual $\pi=\Delta p$ denota a taxa de inflação, $p$ o índice de preços ao consumidor (IPCA, dezembro de $1993=100), y^{r}$ o produto interno bruto dessazonalizado e deflacionado pelo IPCA (média de $1990=100$ ), $m$ o estoque nominal de moeda (conceito M3, em milhares de reais), $R^{d}$ a taxa mensal de juros SELIC, e $R^{b}$ a taxa anual 
de juros de 3 meses (swap pré-DI, 63 dias). ${ }^{2}$ As figuras 2 a 6 ilustram a evolução mensal dessas variáveis entre novembro de 1994 e fevereiro de 2001.

\section{Figura 1}

Principais etapas da modelagem econométrica

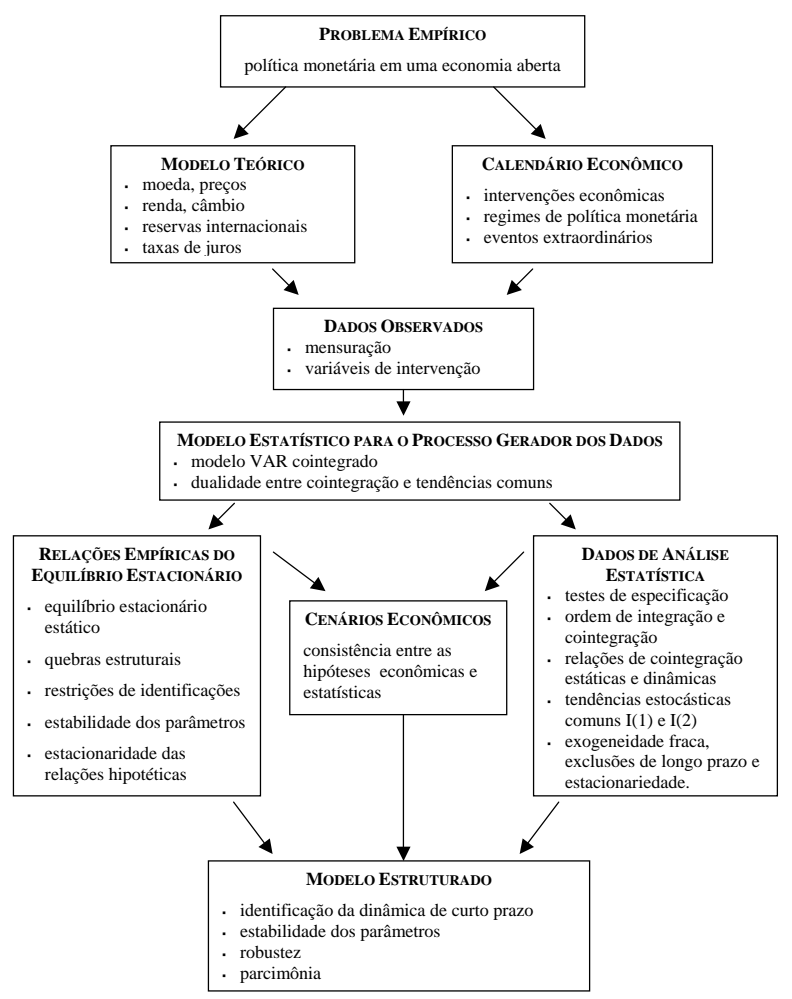

\footnotetext{
${ }^{2}$ Coletamos todas as séries no banco de dados do IPEA (www.ipeadata.gov.br), com exceção das séries referentes às taxas de juros de curto e longo prazo, que foram gentilmente cedidas por Carlos Hamilton V. Araújo (Banco Central do Brasil).
} 
Figura 2

Nível e variação do nível de atividade
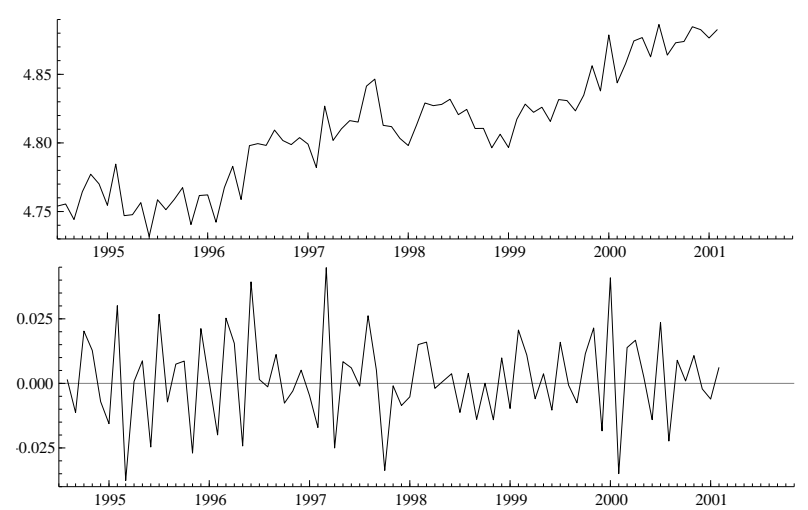

Figura 3

Estoque de moeda nominal e real (nível e variação)
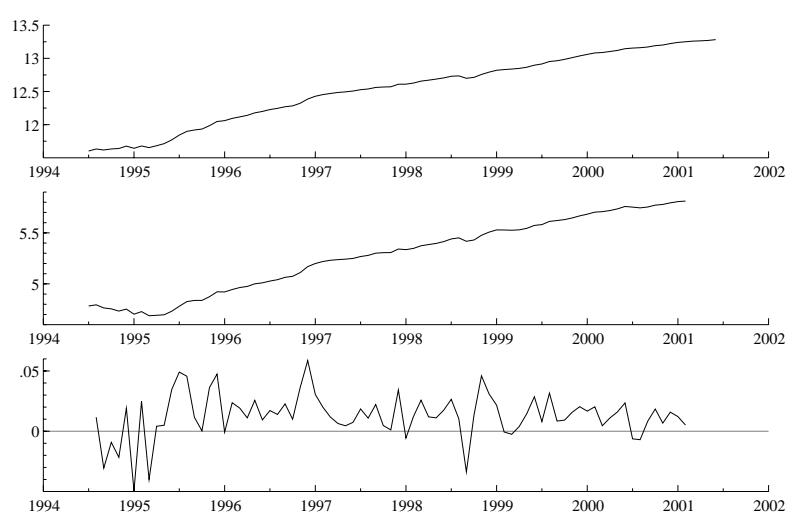
Figura 4

Nível de preços, inflação e variação da inflação
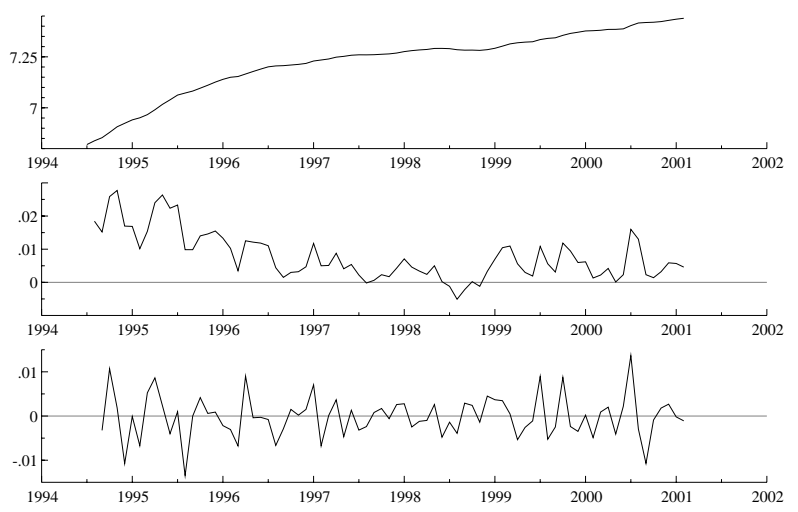

Figura 5

Nível e variação da taxa SELIC

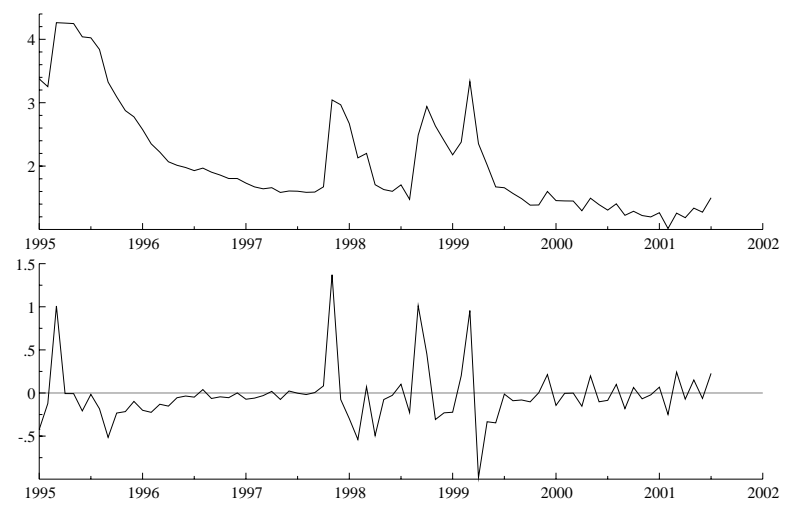


Figura 6

Nível e variação da taxa de juros de 3 meses

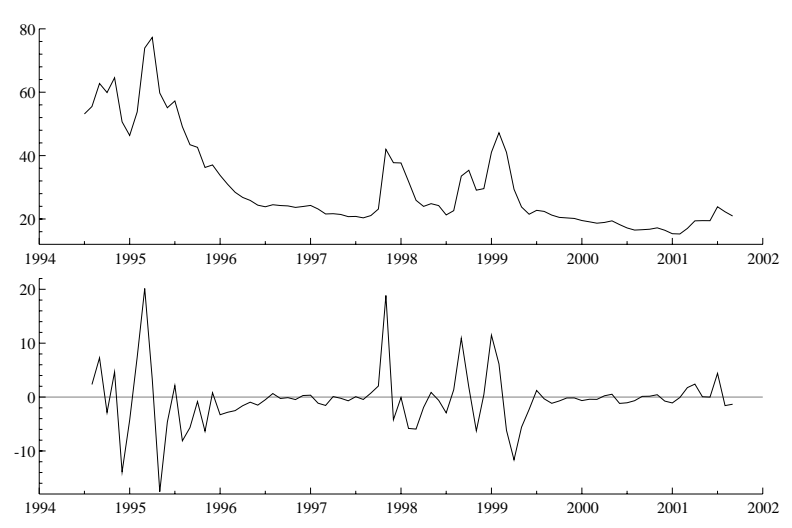

Os resultados da tabela 1 mostram que todas as séries são aparentemente $I(1)$, inclusive o nível de inflação. O nível de preços segue, portanto, um processo $I(2){ }^{3}$ A análise de cointegração na subseção 3.3 confirma a presença de raiz unitária em todas as séries, corroborando nossa estratégia empírica de, na dúvida, tratar as séries como processos integrados (ver a excelente discussão metodológica em Juselius (1999)). Finalmente, a teoria monetária prevê que o nível de preços é relacionado com $m_{t}-y_{t}^{r}$, isto é, com a expansão monetária em excesso ao crescimento do produto real. Como $m_{t}-p_{t}-y_{t}^{r} \sim I(1)$, espera-se que exista uma fator de correção da taxa de inflação aos desvios dessa relação de equilíbrio. Os resultados documentados na seção 4 mostram que este é realmente o caso.

\footnotetext{
${ }^{3}$ Isso implica que não existe nenhum padrão estatisticamente significante de reversão linear à média no período pós-Plano Real, embora nada garanta que a inflação seja estruturalmente $I(1)$. Os resultados de testes de raiz unitária apenas indicam o tratamento assintótico mais apropriado para as séries na amostra.
} 
Tabela 1

Teste KPSS de raiz unitária

\begin{tabular}{cccccr}
\hline Defasagem & $\pi$ & $y^{r}$ & $m-p$ & $R^{d}$ & $R^{b}$ \\
\hline 0 & 2,7839 & 0,7933 & 7,5046 & 3,8921 & 3,4632 \\
1 & 1,6263 & 0,4754 & 3,8180 & 2,0661 & 1,8467 \\
2 & 1,2138 & 0,3634 & 2,5808 & 1,4440 & 1,2992 \\
3 & 0,9942 & 0,3187 & 1,9622 & 1,1285 & 1,0185 \\
4 & 0,8528 & 0,3023 & 1,5913 & 0,9360 & 0,8460 \\
\hline \multicolumn{6}{c}{ Valores críticos assintóticos } \\
\hline \multicolumn{5}{c}{$5 \%$} & 0,463 \\
\hline
\end{tabular}

A primeira coluna indica o número de defasagens do estimador de Newey-West da matriz de covariância de longo prazo da estatística de teste.

\section{Modelo Estatístico}

O modelo vetor auto-regressivo (VAR) de base consiste em

$$
X_{t}=\Pi_{1} X_{t-1}+\ldots+\Pi_{k} X_{t-k}+\Phi D_{t}+\mu+\varepsilon_{t},
$$

no qual $\varepsilon_{t} \sim \operatorname{iid} N(0, \Sigma), X_{t}$ é o vetor de variáveis endógenas do sistema, $\mu$ é um termo determinístico envolvendo apenas uma constante, e $D_{t}$ é um vetor de variáveis binárias capturando intervenções de natureza pontual, transitória, e/ou permanente. Podemos, então, escrever sua representação correção de erros (VECM) como

$$
\Delta X_{t}=\sum_{i=1}^{k-1} \Gamma_{i} \Delta X_{t-i}+\Pi X_{t-1}+\Phi D_{t}+\mu+\varepsilon_{t}
$$

em que $\Pi=\sum_{i=1}^{k-1} \Pi_{i}-I_{k}, \Gamma_{i}=-\sum_{j=i+1}^{k} \Pi_{j}$ e $I_{k}$ é a matriz de identidade de dimensão $k \times k$. O vetor de parâmetros $\Theta=\Gamma_{1}, \ldots, \Gamma_{k-1}, \Pi, \Phi, \mu, \Sigma$ é restrito de tal sorte que a constante $\mu$ pertença ao subespaço linear gerado pelo vetor de cointegração.

Na presença de cointegração, apesar de $\Pi=\alpha \beta^{\prime}$ ter posto reduzido, $\alpha_{\perp}^{\prime} \Gamma \beta_{\perp}$ possui posto pleno para $\Gamma=I_{k}-\sum_{i=1}^{k-1} \Gamma_{i}$. Portanto, a representação vetor média móvel de (4) é

$$
X_{t}=C \sum_{i=1}^{t} \epsilon_{i}+C \mu t+C \Phi \sum_{i=1}^{t} D_{i}+C^{*}(L)\left(\varepsilon_{t}+\mu+\Phi D_{t}\right)+B
$$


na qual $C=\beta_{\perp}\left(\alpha_{\perp}^{\prime} \Gamma \beta_{\perp}\right)^{-1} \alpha_{\perp}^{\prime}, C^{*}(L)$ é um polinômio infinito no operador $L$ de defasagens, e $B$ é uma constante que depende dos valores iniciais (ver Engle e Granger (1987), Hansen (2005)).

\subsection{Estratégia empírica}

As raízes do polinômio característico do modelo VAR possuem uma informação extremamente útil na presença de componentes $I(2)$ ou próximos a $I(2)$. Afinal, há uma raiz unitária adicional para cada tendência $I(2)$ no modelo. Apenas quando não existem componentes $I(2)$ nos dados é que o número de raízes unitárias (ou próximas a raízes unitárias) no polinômio característico será igual ao número de autovalores nulos da matriz $\Pi \equiv \alpha \beta^{\prime}$. A intuição desse resultado reside no fato que $\Gamma$ também contém informação valiosa sobre as raízes unitárias do sistema. As ordens de integração e cointegração podem ser formalmente testadas a partir do procedimento de verossimilhança, apesar da aproximação assintótica ser um pouco suspeita devido ao pequeno número de observações.

A maioria dos modelos empíricos é calcada na análise do estoque real de moeda, assumindo implicitamente que a propriedade de homogeneidade dos preços vale no longo prazo. Nesse contexto, transformar uma variável nominal em real elimina a tendência $I(2)$ presente no estoque nominal de moeda e nos preços, evitando assim a complexidade do modelo $I(2)$. Como encontramos evidências de apenas uma raiz unitária no estoque real de moeda, seguimos a análise empírica com base no modelo $I(1)$ assumindo que, no longo prazo, vale a propriedade de homogeneidade dos preços.

\subsection{Modelo empírico}

Estimamos um modelo VAR com constante restrita ao espaço de cointegração motivados pela relação (2) entre a pressão de demanda e a curva de Phillips. O vetor de variáveis endógenas consiste em $X_{t}^{\prime}=\left(\pi_{t}, y_{t}^{r}, m_{t}-p_{t}, R_{t}^{d}, R_{t}^{b}\right)$, em que $\pi_{t}=\Delta p_{t}$ denota a taxa de inflação, $p_{t}$ o índice de preços ao consumidor (IPCA), $y_{t}^{r}$ o produto interno bruto dessazonalizado e deflacionado pelo IPCA, $m_{t}$ o estoque nominal de moeda (conceito M3), $R_{t}^{d}$ a taxa de juros SELIC, e $R_{t}^{b}$ a taxa de juros de 3 meses (swap pré-DI, 63 dias). O modelo inclui três variáveis binárias de intervenção relacionadas às crises da Rússia e da Ásia, e à desvalorização do real no início de 1999 (ver figura 7). Consideramos, ainda, a variação mensal das reservas internacionais (conceito liquidez internacional, em milhões de dólares) e a variação mensal da taxa de câmbio (ver figura 8). Assumimos que essas duas 
variáveis são exógenas, uma vez que suas variações neste período foram, em grande parte, causadas por fenômenos não pertinentes ao sistema.

Figura 7

Variáveis de intervenção

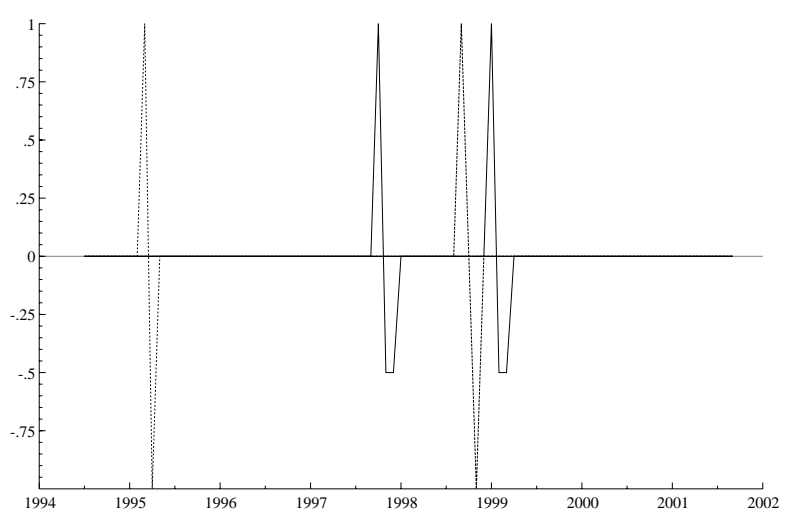

Figura 8

Variáveis exógenas
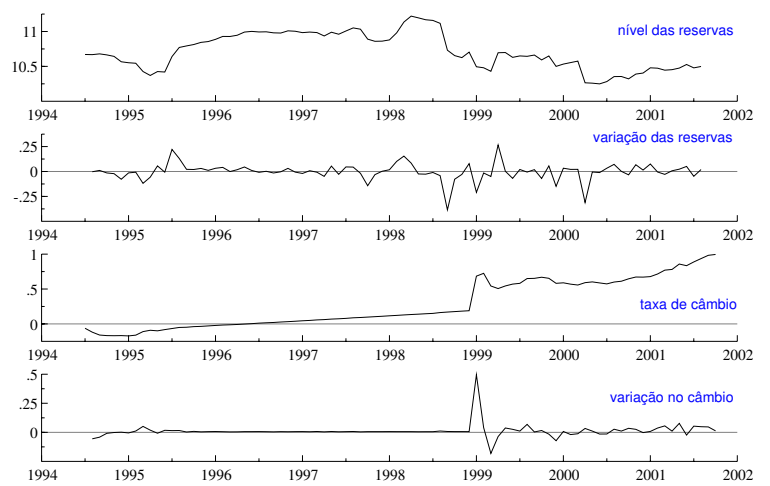
Tabela 2

Seleção da estrutura de defasagens

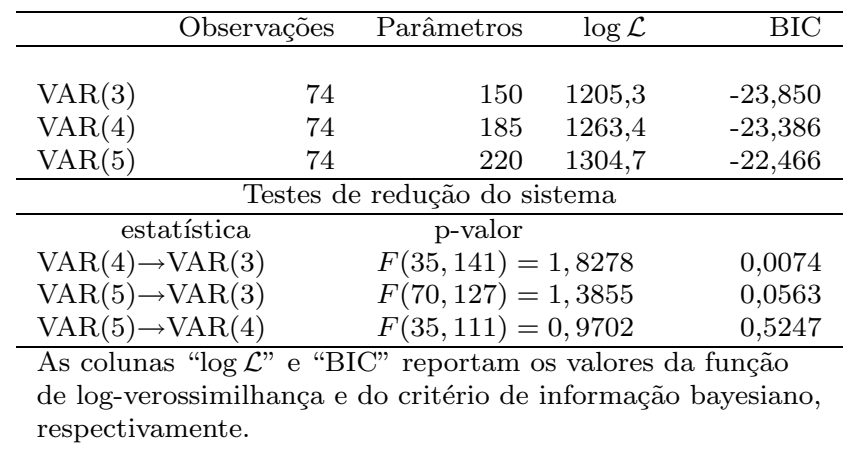

A tabela 2 reporta os resultados do processo de seleção da ordem do VAR. Iniciamos o processo com um modelo VAR(5) por encerrar uma estrutura suficientemente rica de defasagens. Estimamos adicionalmente modelos $\operatorname{VAR}(k)$, $k \in 1, \ldots, 4$ e comparamos os resultados através do critério de informação bayesiano e de testes-F de redução da ordem auto-regressiva. Após descartamos os modelos $\operatorname{VAR}(1)$ e $\operatorname{VAR}(2)$ por apresentarem autocorrelação residual, verificamos que o critério de informação sugere uma especificação $\operatorname{VAR}(3)$. Os resultados dos testes-F corroboram parcialmente esta evidência. Afinal, para controlar a acumulação do erro do tipo I, precisamos considerar níveis decrescentes de significância na seqüência de testes-F (ver Terasvirta e Mellin (1986)). ${ }^{4}$

Temos, então, o seguinte modelo estatístico

$$
\Delta X_{t}=\Gamma_{1} \Delta X_{t-1}+\Gamma_{2} \Delta X_{t-2}+\alpha \beta^{\prime} X_{t-1}+\sum_{i=0}^{3} \Psi_{i} Z_{t-i}+\Phi, D_{t}+\mu+\varepsilon_{t}
$$

no qual $\varepsilon_{t} \sim \operatorname{iid} N(0, \Sigma), Z_{t}$ denota o vetor de variáveis exógenas e $D_{t}$ inclui as variáveis binárias de intervenção referentes às crises asiática, russa e brasileira. A inclusão de variáveis exógenas estacionárias no modelo VAR cointegrado pode trazer um problema de parâmetros de estorvo na distribuição assintótica do teste do traço. Para evitar esse tipo de problema, seguimos a recomendação de Rahbek e Mosconi (1999) e acumulamos as variáveis exógenas antes de incorporá-las ao termo de equilíbrio de longo prazo. A representação VECM do modelo consiste, então, em

\footnotetext{
${ }^{4} \mathrm{O}$ procedimento padrão considera um nível de significância $\alpha^{i}$ para o $i$-ésimo teste.
} 


$$
\begin{aligned}
\Delta X_{t} & =\Gamma_{1} \Delta X_{t-1}+\Gamma_{2} \Delta X_{t-2}+\alpha\left(\beta^{\prime} X_{t-1}+\beta^{\prime} \sum_{i=i}^{t} Z_{i}\right) \\
& +\Upsilon_{0} Z_{t}+\sum_{i=1}^{2} \Upsilon_{i} \Delta Z_{t-i}+\Phi D_{t}+\mu+\varepsilon_{t},
\end{aligned}
$$

em que os parâmetros $\Upsilon_{0}, \Upsilon_{1}$ e $\Upsilon_{2}$ são funções do vetor de parâmetros $\left(\Psi_{0}, \Psi_{1}, \Psi_{2}, \Psi_{3}\right)$.

Os resultados empíricos sugerem que o VAR irrestrito explica a maior parte da variação dos dados, uma vez que os coeficientes $R^{2}$ de determinação são bastante elevados. Para verificar a adequação do modelo, efetuamos uma série de testes de especificação (ver tabela 3). A possível presença de efeitos ARCH nos resíduos da taxa SELIC não preocupa muito, pois a análise de cointegração costuma ser robusta à presença de heterocedasticidade condicional (Gozalo, 1994, Silvapulle e Podivinsky, 2000, Rahbek et alii, 2002). A ausência de autocorrelação residual e a normalidade dos resíduos garantem uma situação confortável para realizar a análise de cointegração na próxima seção.

\section{Tabela 3}

\begin{tabular}{|c|c|c|c|c|c|}
\hline & \multicolumn{5}{|c|}{ Testes multivariados } \\
\hline \multirow{4}{*}{$\begin{array}{l}\text { Autocorrelação } \\
\text { Normalidade }\end{array}$} & \multirow{2}{*}{\multicolumn{3}{|c|}{$\begin{array}{l}F(100,107)=1,1938 \\
\chi^{2}(10)=11,19\end{array}$}} & \multirow{2}{*}{\multicolumn{2}{|c|}{$\begin{array}{l}\text { p-valor }=0,1838 \\
\text { p-valor }=0,3429\end{array}$}} \\
\hline & & & & & \\
\hline & \multicolumn{5}{|c|}{ Testes univariados, p-valores } \\
\hline & $\Delta \pi$ & $\Delta y^{r}$ & $\Delta(m-p)$ & $\Delta R^{d}$ & $\Delta R^{b}$ \\
\hline $\operatorname{arch}(2)$ & 0,9070 & 0,7921 & 0,8538 & 0,0290 & 0,2452 \\
\hline autocorrelação & 0,115 & 0,793 & 0,390 & 0,619 & 0,1295 \\
\hline$\hat{\sigma}_{\varepsilon}$ & 0,0036 & 0,0130 & 0,0165 & 0,1900 & 3,587 \\
\hline
\end{tabular}

Testes de especificação, VAR irrestrito 


\subsection{Propriedades de cointegração}

Para investigar as relações de longo prazo discutidas na subseção 2.1, examinamos algumas especificações alternativas. Em particular, consideramos hipóteses da forma $\beta=\left\{H \phi_{1}, \psi_{1}, \psi_{2}\right\}$ para testar individualmente cada relação de longo prazo. O objetivo destes testes é fornecer informação sobre a relação direta entre os instrumentos de política monetária e suas metas. Por exemplo, se uma expansão monetária sempre gera inflação, então podemos esperar que exista um vetor de cointegração relacionando positivamente a inflação esperada e a velocidade da moeda. Como expansão monetária também pode ser inflacionária por aumentar as pressões do lado da demanda, a transmissão monetária pode ser via uma cointegração positiva entre inflação e hiato do produto.

Tabela 4 apresenta uma série de hipóteses sobre as relações de longo prazo. $\mathcal{H}_{1}$ procura estimar a demanda por moeda. Os coeficientes das taxas de juros indicam que os agentes demandam mais moeda quando o retorno de ativos alternativos à moeda diminui. $\mathcal{H}_{2}$ procura identificar uma função de reação do Banco Central centrada no controle da taxa SELIC. $\mathcal{H}_{3}$ avalia a relação de longo prazo entre o hiato do produto e a taxa de juros de 3 meses.

As demais hipóteses testam relações de longo prazo alternativas entre as taxas de juros (instrumento e alvo intermediário do Banco Central) e a inflação (meta do Banco Central). $\mathcal{H}_{4}$ testa a estacionariedade da diferença entre as taxas de juros de curto e longo prazo. $\mathcal{H}_{5}$ testa se existe alguma combinação linear entre as taxas de juros de curto e longo prazo que seja estacionária. $\mathcal{H}_{6}$ e $\mathcal{H}_{7}$ verificam se alguma taxa de juros real é estacionária. Finalmente, $\mathcal{H}_{8}$ testa se a diferença entre as taxas de juros de curto e longo prazo cointegram com a inflação. Os resultados mostram que todas essas relações de longo prazo alternativas são rejeitadas empiricamente. 
Tabela 4

Relações de longo prazo

\begin{tabular}{|c|c|c|c|c|c|c|c|c|c|c|c|c|}
\hline & $\pi$ & $y^{r}$ & $m-p$ & $R^{d}$ & $R^{b}$ & Tendência & Ásia & Rússia & Brasil & Câmbio & Reservas & $\mathrm{P}$-valor \\
\hline $\mathcal{H}_{1}$ & & $-15,471$ & 1,0000 & 1,5133 & $-0,098525$ & 0,0267 & $-0,34359$ & $-1,3732$ & 1,0135 & & & 0,864 \\
\hline $\mathcal{H}_{2}$ & $-117,68$ & 1,0000 & & 1,0000 & & $-0,0009$ & $-0,794$ & $-0,88762$ & 0,2061 & 2,665 & $-0,371$ & 0,176 \\
\hline $\mathcal{H}_{3}$ & 1,665 & 1,0000 & & & 0,0015 & $-0,0009$ & $-0,004$ & 0,05481 & $-0,024$ & $-0,013$ & 0,055 & 0,921 \\
\hline $\mathcal{H}_{4}$ & & & & 1,0000 & $-1,0000$ & & & & & & & 0,052 \\
\hline $\mathcal{H}_{5}$ & & & & $-2,245$ & 0,195 & 0,015 & & & & & & 0,133 \\
\hline $\mathcal{H}_{6}$ & $-81,553$ & & & 1,0000 & & & & & & & & 0,069 \\
\hline $\mathcal{H}_{7}$ & $-1389,9$ & & & & 1,0000 & & & & & & & 0,517 \\
\hline $\mathcal{H}_{8}$ & 1308,1 & & & 1,0000 & $-1,0000$ & & & & & & & 0,140 \\
\hline
\end{tabular}

correspondem às variáveis de intervenção associadas aos respectivos períodos de crise. 


\subsection{Identificação estrutural das relações de longo prazo}

A hipótese de uma estrutura de cointegração completamente identificada pode ser expressa como $\beta_{r}=\left\{H_{1} \phi_{1}, H_{2} \phi_{2}, H_{3} \phi_{3}\right\}$, em que as matrizes $H_{i}(i=1,2,3)$ impõem restrições em cada vetor de cointegração. O teste de sobre-identificação dessas restrições é calcado na estatística de razão de verossimilhança (RV) e possui uma distribuição limite $\chi^{2}(v)$, na qual o número de graus de liberdade $v$ coincide com o número de restrições de sobre-identificação (Johansen e Juselius, 1994).

A tabela 5 apresenta os três vetores de cointegração identificados. Para avaliar o número de relações de cointegração, consideramos não apenas o teste do traço, ${ }^{5}$ mas também as próprias estimativas dos autovalores e uma análise gráfica das relações de longo prazo. A figura 9 ilustra o comportamento dos três vetores de cointegração ao longo do período.

Figura 9

vetores de cointegração
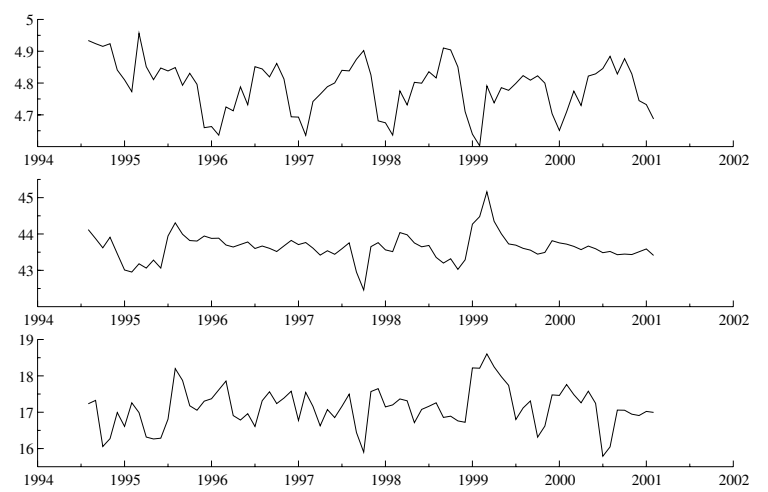

\footnotetext{
${ }^{5}$ Como o número de graus de liberdade do teste do traço em pequenas amostras é reduzido, sua aproximação assintótica não é necessariamente satisfatória. Infelizmente, não existe ainda nenhum método estatístico que assegure uma correção satisfatória para esse problema, apesar de algumas tentativas muito interessantes à nível teórico (Johansen, 2000, 2005).
} 
Tabela 5

Estrutura identificada de longo prazo

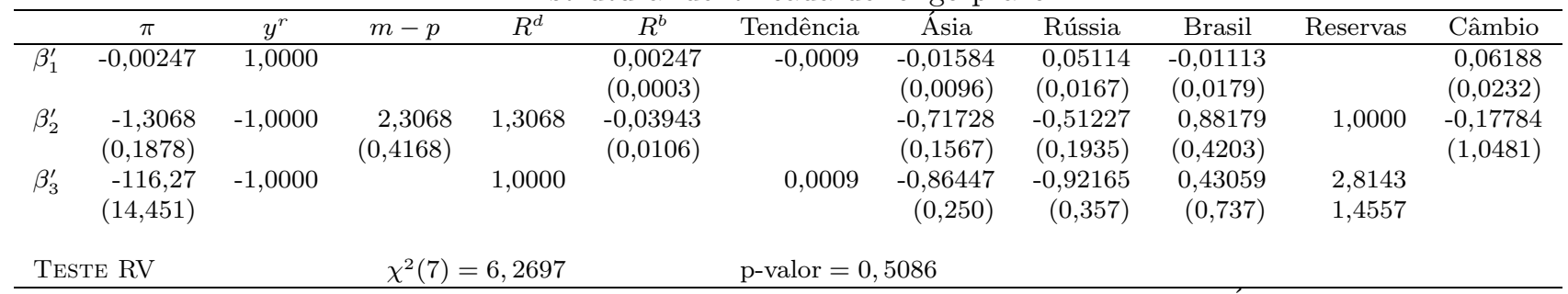

A coluna "tendência" apresenta o coeficiente associado à tendência linear, enquanto que as colunas "Ásia", "Brasil" e "Rússia" correspondem às variáveis de intervenção associadas aos respectivos períodos de crise. Os valores em parênteses referem-se aos respectivos erros padrão. 
A primeira relação $\hat{\beta}_{1}^{\prime} X_{t}$ estabelece uma relação negativa entre o hiato do produto real e a taxa de juros real, conforme a curva IS em (2). A interpretação da segunda relação de longo prazo $\hat{\beta}_{2}^{\prime} X_{t}$ é um pouco menos evidente. Em uma primeira análise, consideramos que estivesse relacionada à demanda por moeda descrita em (1). Entretanto, como o sinal do coeficiente associado à inflação não correspondia ao seu efeito esperado, passamos a interpretá-la como uma equação de determinação do nível de reservas, conforme sugestão de um parecerista anônimo.

Esta dificuldade de interpretação provavelmente sinaliza que o ambiente inflacionário presente na primeira parte da amostra conecta a demanda por moeda local à determinação do nível de reservas devido ao processo de substituição de moedas. Para verificar esta possibilidade, quando identificamos o espaço de cointegração, permitimos que a taxa de juros de longo prazo entrasse na segunda relação. Interessantemente, os vetores de cointegração quase não se alteram, além de conservarem os sinais esperados. A nossa interpretação é consistente com os modelos monetários de câmbio (ver, entre outros, Frenkel (1976), Mussa (1976)). Em particular, uma taxa de inflação mais alta induz um processo de substituição de moedas e, conseqüentemente, um crescimento no volume de ativos estrangeiros em poder dos residentes locais. Por outro lado, uma maior demanda por reais implica uma redução na demanda por reservas devido ao efeito substituição (realocação da carteira de ativos locais). Por sua vez, um aumento da taxa de juros de longo prazo gera um maior nível de reservas por tornar os ativos locais mais atrativos para os investidores estrangeiros. Finalmente, a relação negativa entre os movimentos da taxa SELIC e o volume de reservas aponta para a incapacidade de conter o fluxo de reservas via ajustes na taxa de curto prazo.

O terceiro vetor de cointegração $\hat{\beta}_{3}^{\prime} X_{t}$ determina, por sua vez, uma relação de homogeneidade entre as taxas de juros de curto e longo prazo. A taxa de juros SELIC responde às pressões inflacionárias, ao hiato do produto real e às variações das reservas internacionais. Essa última relação pode ser interpretada como a regra de reação monetária utilizada pelo Banco Central para definir a taxa SELIC, como explicitado em (3). O sinal do coeficiente associado à inflação sugere que a taxa de inflação serve como uma proxy do prêmio de risco aliado ao preço relativo dos juros de longo e curto prazo. Finalmente, é interessante observar que, apesar das mudanças aparentes na condução da política monetária no período considerado, as estimativas recursivas dos vetores de cointegração indicam que os parâmetros são bastante estáveis. 


\section{Ajustes de Curto Prazo}

A identificação dos ajustes de curto prazo pode ser feita a partir da representação VECM do modelo VAR condicionando o modelo nos vetores de cointegração identificados. A estrutura proposta a seguir resulta de um procedimento de seleção de modelos partindo do geral para o específico, em que os coeficientes estatisticamente iguais a zero são seqüencialmente eliminados desde que não haja autocorrelação residual na especificação resultante. Os coeficientes associados ao ajuste de curto prazo às relações de longo prazo não apenas são significativos em praticamente todas as equações, como também possuem o sinal esperado. Portanto, a combinação linear $\hat{\beta}_{r}^{\prime} X_{t}$ pode ser interpretada como o vetor dos equilíbrios estacionários do sistema (Johansen, 2005).

O sistema final de equações é dado por

$$
\begin{aligned}
& \Delta \pi_{t}=\underset{0,0739}{0,2167}+\underset{0,0998}{0,2987} \Delta \pi_{t-1}-\underset{0,0068}{0,0049} \Delta y_{t-1}^{r}+\underset{0,0007}{0,0012} r_{t-2}+\underset{0,0140}{0,0267} \Delta e_{t-2} \\
& +\underset{0,0065}{0,0116} \hat{\beta}_{1}^{\prime} X_{t-1}-\underset{0,0016}{0,0970} \hat{\beta}_{2}^{\prime} X_{t-1}+\underset{0,0012}{0,0089} \hat{\beta}_{3}^{\prime} X_{t-1}+\varepsilon_{t}^{(\pi)} \\
& \Delta y_{t}^{r}=\underset{0,3884}{2,1755}-\underset{1,1409}{2,7235} \Delta \pi_{t-2}+\underset{0,0881}{0,2859} \Delta y_{t-2}^{r}-\underset{0,3051}{0,8869} \Delta(m-p)_{t-2} \\
& -\underset{0,019}{0,017} \Delta R_{t-1}^{d}-\underset{0,0047}{0,0091} r_{t-2}+\underset{0,0388}{0,0942} \mathrm{Asia}_{t}-\underset{0,0719}{0,4990} \hat{\beta}_{1}^{\prime} X_{t-1}+\varepsilon_{t}^{(y)}(8) \\
& \Delta(m-p)_{t}=\underset{0,0022}{0,0104}+\underset{0,3825}{0,7077} \Delta \pi_{t-2}+\underset{0,0969}{0,2683} \Delta(m-p)_{t-2} \\
& -\underset{0,0003}{0,0009} \Delta R_{t-2}^{b}+\underset{0,0014}{0,0025} r_{t-1}-\underset{0,0106}{0,0496 \text { Russia }_{t}}+\varepsilon_{t}^{(m-p)} \\
& \Delta R_{t}^{d}=\underset{2,4700}{28,235} \underset{0,2600}{0,8704} \Delta y_{t-2}^{r}+\underset{1,0660}{3,9110} \Delta(m-p)_{t-1}-\underset{0,0771}{0,2678} \Delta R_{t-1}^{d} \\
& -\underset{0,0735}{0,3626} \Delta R_{t-2}^{d}+\underset{0,0044}{0,0216} \Delta R_{t-1}^{b}+\underset{0,0041}{0,0187} \Delta R_{t-2}^{b}-\underset{0,0325}{0,1431} r_{t-1} \\
& -\underset{0,0360}{0,2152} r_{t-2}-\underset{0,6640}{2,6509} \Delta e_{t-1}-\underset{0,6990}{2,4609} \Delta e_{t-2}-\underset{0,1339}{0,4155} \text { Asia }_{t}
\end{aligned}
$$

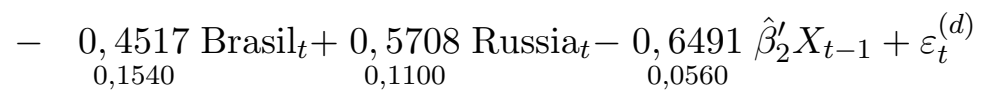




$$
\begin{aligned}
& \Delta R_{t}^{b}=\underset{58,38}{4} \underset{69,52}{48,55}-\underset{72,18}{154,86} \Delta \pi_{t-1}-\underset{23,500}{299,77} \Delta \pi_{t-2}+\underset{76,514}{62} \Delta(m-p)_{t-1}
\end{aligned}
$$

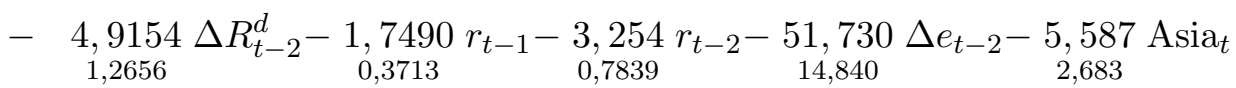

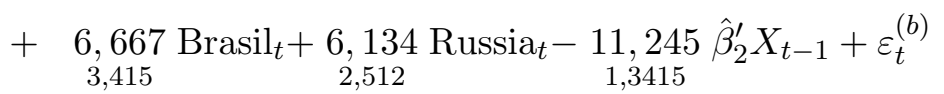

em que $\varepsilon_{t}^{(\cdot)}$ denota o termo residual de cada equação e os valores em fonte miúda correspondem aos erros padrão das estimativas dos coeficientes. A figura 10 exibe a boa aderência do modelo contrastando os valores real e estimado para as cinco variáveis.

A tabela 6 agrupa os resultados dos testes de diagnósticos para o modelo VECM restrito. Os testes multivariados apresentam resultados um pouco diferentes àqueles obtidos para os resíduos do modelo VAR irrestrito. A hipótese de normalidade vetorial é facilmente rejeitada devido ao excesso de curtose presente nas taxas de juros. Em contraste, os resultados dos testes univariados são bastante semelhantes aos da tabela 3 . A única diferença reside no teste de heterocedasticidade condicional, que dessa vez acusa problemas apenas no resíduo $\hat{\varepsilon}_{t}^{(b)}$ correspondente à taxa SELIC. Esses resultados não invalidam os anteriores, uma vez que a análise de cointegração é robusta ao excesso de curtose e à heterocedasticidade condicional (Gozalo, 1994, Silvapulle e Podivinsky, 2000, Rahbek et alii, 2002).

Tabela 6

Testes de especificação, VECM restrito

\begin{tabular}{|c|c|c|c|c|c|}
\hline & \multicolumn{5}{|c|}{ Testes multivariados } \\
\hline \multirow{4}{*}{$\begin{array}{l}\text { Autocorrelação } \\
\text { normalidade }\end{array}$} & \multirow{2}{*}{\multicolumn{4}{|c|}{$\begin{array}{l}F(50,245)=1,2104 \\
\chi^{2}(10)=32,358\end{array}$}} & p-valor $=0,1755$ \\
\hline & & & & & $\mathrm{p}$-valor $=0,0003$ \\
\hline & \multicolumn{5}{|c|}{ Testes univariados, p-valores } \\
\hline & $\Delta \pi$ & $\Delta y^{r}$ & $\Delta(m-p)$ & $\Delta R^{d}$ & $\Delta R^{b}$ \\
\hline $\operatorname{arch}(2)$ & 0,9202 & 0,9805 & 0,1311 & 0,3729 & 0,0208 \\
\hline Autocorrelação & 0,9943 & 0,9994 & 0,9848 & 0,1396 & 0,5303 \\
\hline$\hat{\sigma}_{\varepsilon}$ & 0,00355 & 0,04973 & 0,0158 & 0,1634 & 3,49 \\
\hline
\end{tabular}

Os testes de autocorrelação verificam a presença de correlação serial de segunda ordem nos resíduos.

A tabela 7 reporta a matriz de correlação estimada dos resíduos do modelo VECM restrito. O sistema de equações (7)-(11) é praticamente suficiente para explicar as relações contemporâneas entre as variáveis. Excetuando a correlação positiva residual entre as taxas de juros de curto e longo prazo, os termos fora 
da diagonal principal são estatisticamente próximos de zero. Apesar disso, não podemos dar uma interpretação realmente estrutural às relações de curto prazo e, portanto, debater as relações contemporâneas entre os instrumentos de política monetárias e as demais variáveis. Destacamos, então, apenas alguns aspectos interessantes nos ajustes às relações de equilíbrio de longo prazo, pois possuem informação relevante sobre a dinâmica do sistema e interessantes interpretações econômicas.

Tabela 7

Matriz de correlação dos resíduos

\begin{tabular}{lrrrrr}
\hline \multicolumn{1}{c}{$\pi$} & $y^{r}$ & $m-p$ & $R^{d}$ & $R^{b}$ \\
\hline & $\pi$ & 1,0000 & & & \\
$y^{r}$ & $-0,0763$ & 1,0000 & & & \\
$m-p$ & $-0,2664$ & $-0,2433$ & 1,0000 & & \\
$R^{d}$ & $-0,0208$ & 0,4243 & $-0,1827$ & 1,0000 & \\
$R^{b}$ & 0,1353 & 0,0816 & $-0,3645$ & 0,5737 & 1,0000 \\
\hline
\end{tabular}

Primeiro, a inflação apresenta um ajuste significativo de curto prazo a todas as relações de longo prazo, sugerindo a viabilidade do regime de metas inflacionárias recentemente adotado pelas autoridades monetárias brasileiras. A taxa SELIC se ajusta a desequilíbrios na equação de determinação do nível de reservas internacionais. A correção tem o sinal apropriado e corrobora a evidência que a determinação das reservas se dá, no curto prazo, através de alterações na taxa SELIC. Segundo, a função de reação de longo prazo do Banco Central afeta diretamente, no curto prazo, apenas a taxa de inflação, porém não há correção de erros. Os movimentos da taxa SELIC são consistentes com o status de principal instrumento monetário do Banco Central brasileiro, pois não há reação estatisticamente significativa à regra de reação monetária do Banco Central.

Terceiro, o estoque real de moeda é fracamente exógeno em relação aos vetores de cointegração e, portanto, constitui uma tendência estocástica comum do sistema. ${ }^{6}$ Este resultado evidencia a passividade da atual política monetária brasileira no que tange os fluxos de moeda, corroborando a conjectura que o Banco Central altera a taxa de juros de curto prazo objetivando evitar flutuações indesejadas nos fluxos de capitais. Ademais, as estimativas corroboram a identificação da taxa de juros de 3 meses como meta intermediária dado seus ajustes à taxa SELIC e ao excesso de moeda.

Para confirmar as nossas interpretações das relações de curto prazo, condu-

${ }^{6}$ A validade desta asserção independe do processo de identificação estrutural de curto prazo. Exogeneidade fraca em relação aos vetores de cointegração exige apenas que o coeficiente de ajuste às relações de longo prazo seja zero (Johansen (1995), Teorema 8.1, pág. 122). 
zimos uma análise de impulso-resposta para avaliar a reação do sistema a um choque monetário. A matriz de correlação dos resíduos na tabela 7 sugere uma decomposição de Choleski mantendo a própria ordem das variáveis: $\left(\pi, y^{r}, m-\right.$ $\left.p, R^{d}, R^{b}\right)$. A figura 11 ilustra, então, a resposta do sistema (7)-(11) a um choque monetário de um desvio padrão na taxa de juros SELIC de curto prazo. A dinâmica de resposta segue o padrão clássico de transmissão monetária. A elevação na taxa de juros de curto prazo gera, inicialmente, um aumento da taxa de juros de longo prazo e um efeito negativo nas demais variáveis. Os efeitos do choque monetário tornam-se nulos após 6 meses, entretanto. Finalmente, vale observar que o nível de atividade reage de forma consistente com a interpretação usual de choque monetário (ausência de efeito real de longo prazo). Após a queda inicial, há uma rápida recuperação do nível de atividade, tornando o efeito acumulado do choque monetário praticamente nulo.

Figura 10

Valor real $\mathrm{x}$ valor estimado
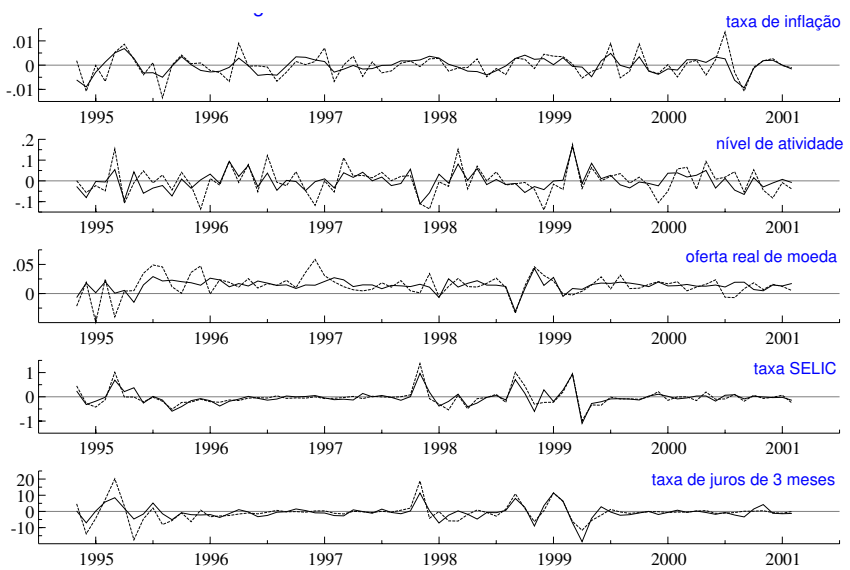
Figura 11

Resposta do sistema a um choque monetário
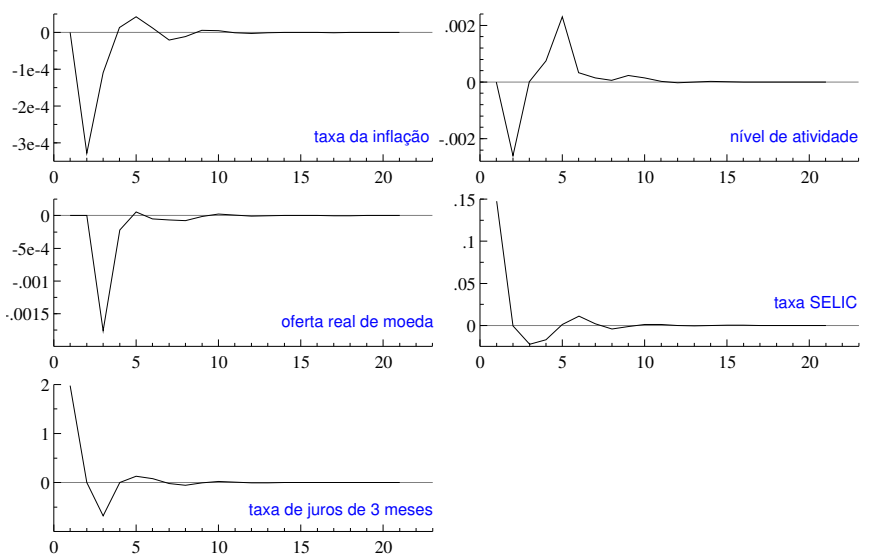

\section{Conclusão}

Este trabalho estima o mecanismo de transmissão monetária da economia brasileira pós-Plano Real via um modelo VAR cointegrado para o estoque real de moeda, renda real agregada, inflação, e taxas de juros de curto e longo prazo. Para dar uma conotação estrutural ao sistema dinâmico estimado, identificamos os vetores de cointegração a partir de relações econômicas de equilíbrio. Os resultados empíricos evidenciam que o estoque real de moeda é uma tendência estocástica comum do sistema e que existem três relações de longo prazo.

A primeira corresponde a uma curva IS, estabelecendo uma relação negativa entre o hiato do produto real e a taxa de juros real. O segundo vetor de cointegração determina o nível de reservas em função da velocidade da moeda, da inflação e das taxas de juros de curto e longo prazo. A terceira relação identifica a regra de reação monetária do Banco Central para fixar a taxa de juros SELIC. Finalmente, a dinâmica de curto prazo implícita na representação VECM e a análise de impulso-resposta ilustram a boa aderência do modelo. 


\section{Referências}

Camargo, J. M., Neri, M. C., \& Reis, M. C. (2002). Employment and productivity in Brazil in the nineties. In Bauman, R., editor, Brazil in the 1990s: An Economy in Transition. St. Antony's Series, Palgrave, Cambridge.

Carneiro, D. D. \& Wu, T. Y. H. (2004). Contas externas e política monetária. Revista Brasileira de Economia, 58:301-323.

Cati, R. C., Garcia, M. G. P., \& Perron, P. (1999). Unit roots in the presence of abrupt governmental interventions with an application to Brazilian data. Journal of Applied Econometrics, 14:27-56.

Clarida, R., Galí, J., \& Gertler, M. (1998). Monetary policy rules in practice: Some international evidence. European Economic Review, 42:1033-1067.

Dickey, D. A. \& Fuller, W. A. (1979). Distribution of estimators for autoregressive time series with a unit root. Journal of the American Statistical Association, 74:427-431.

Engle, R. F. \& Granger, C. W. J. (1987). Co-integration and error correction: Representation, estimation and testing. Econometrica, 55:251-276.

Franses, P. H. \& Haldrup, N. (1994). The effects of additive outliers on tests for unit roots and cointegration. Journal of Business and Economic Statistics, 12:471-478.

Frenkel, J. (1976). A monetary approach to the exchange rate: Doctrinal aspects and empirical evidence. Scandinavian Journal of Economics, 78:220-24.

Garrat, A., Lee, K., Pesaran, M. H., \& Shin, Y. (2000). A structural cointegrating VAR approach to macroeconomic modelling. In Holly, S. \& Weale, M., editors, Econometric Modelling: Techniques and Applications. Cambridge University Press, Cambridge.

Gozalo, J. (1994). Five alternative methods of estimating long-run equilibrium relationships. Journal of Econometrics, 60:203-233.

Hansen, P. H. (2005). Granger's representation theorem: A closed-form expression for I(1) processes. Econometrics Journal, 8:23-28.

Johansen, S. (1995). Likelihood-Based Inference in Cointegrated Vector Autoregressive Models. Oxford University Press, Oxford. 
Johansen, S. (2000). A Bartlett correction factor for tests on the cointegrating relations. Econometric Theory, 16:740-778.

Johansen, S. (2005). The interpretation of cointegrating coefficients in the cointegrated vector autoregressive model. Oxford Bulletin of Economics and Statistics, 67:93-104.

Johansen, S. \& Juselius, K. (1994). Identification of the long-run and the shortrun structure: An application to the IS-LM model. Journal of Econometrics, $63: 7-36$.

Juselius, K. (1998). Changing monetary transmission mechanisms within the EU. Empirical Economics, 23:455-481.

Juselius, K. (1999). Models and relations in economics and econometrics. Journal of Economic Methodology, 6:259-290.

Juselius, K. (2001). European integration and monetary transmission mechanisms: The case of Italy. Journal of Applied Econometrics, 16:341-358.

Kwiatkowski, D., Phillips, P. C. B., Schmidt, P., \& Shin, Y. (1992). Testing the null hypothesis of stationarity against the alternative of a unit root. Journal of Econometrics, 54:159-178.

Mussa, M. (1976). The exchange rate, the balance of payments and monetary and fiscal policy under a regime of controlled floating. Scandinavian Journal of Economics, 78:229-48.

Phillips, P. C. B. \& Perron, P. (1988). Testing for a unit root in time series regression. Biometrika, 75:335-346.

Rahbek, A., Hansen, E., \& Dennis, J. G. (2002). ARCH innovations and their impact on cointegration rank testing. Preprint 22, Department of Theoretical Statistics, University of Copenhagen.

Rahbek, A. \& Mosconi, R. (1999). Cointegration rank inference with stationary regressors in VAR models. Econometrics Journal, 2:76-91.

Silvapulle, P. S. \& Podivinsky, J. M. (2000). The effect of non-normal disturbances and conditional heteroskedasticity on multiple cointegration tests. Journal of Statistical Computation and Simulation, 65:173-189.

Terasvirta, T. \& Mellin, I. (1986). Model selection criteria and model selection tests in regression models. Scandinavian Journal of Statistics, 52:159-171.

RBE Rio de Janeiro 59(1):5-32 JAN/MAR 2005 